\title{
Comparison of Complications Following Implant-Based Breast Reconstruction Using Triple Antibiotic Solution versus Low Concentration Chlorhexidine Gluconate Solution
}

\author{
Tyler K. Merceron'1, Udayan Betarbet2, Alexandra Hart³, Nusaiba Baker², Grant Carlson³, \\ Albert Losken ${ }^{3}$
}

${ }^{1}$ Department of Surgery, Emory University School of Medicine, Atlanta, GA, USA

${ }^{2}$ School of Medicine, Emory University, Atlanta, GA, USA

${ }^{3}$ Division of Plastic Surgery, Emory University School of Medicine, Atlanta, GA, USA

Email: alosken@emory.edu

How to cite this paper: Merceron, T.K., Betarbet, U., Hart, A., Baker, N., Carlson, G. and Losken, A. (2019) Comparison of Complications Following Implant-Based Breast Reconstruction Using Triple Antibiotic Solution versus Low Concentration Chlorhexidine Gluconate Solution. Modern Plastic Surgery, 9, 74-85.

https://doi.org/10.4236/mps.2019.94010

Received: September 3, 2019

Accepted: October 12, 2019

Published: October 15, 2019

Copyright $\odot 2019$ by author(s) and Scientific Research Publishing Inc. This work is licensed under the Creative Commons Attribution International License (CC BY 4.0).

http://creativecommons.org/licenses/by/4.0/

\begin{abstract}
Background: Prevention of infection and capsular contracture remains a primary goal of implant-based breast reconstruction (IBBR). Previous studies have demonstrated improved outcomes with the use of triple-antibiotic solution (TAS) for breast pocket irrigation, but ready-to-use products have recently gained popularity. The purpose of this study is to compare outcomes following IBBR between TAS and low-concentration chlorhexidine gluconate (CHG) solutions. Methods: This is a retrospective analysis of 690 consecutive patients undergoing IBBR from 2008-2017. The TAS $(n=346)$ irrigation solution was composed of $1 \mathrm{~g}$ cefazolin, $80 \mathrm{mg}$ gentamicin and 50,000 $\mathrm{U}$ bacitracin diluted in $500 \mathrm{~mL}$ of normal saline; the CHG $(\mathrm{n}=344)$ irrigation solution was the commercially-available product Irrisept $^{\oplus}(0.05 \%$ chlorhexidine gluconate in sterile water, Irrimax Corporation, Lawrenceville, GA). Comparisons were made between demographic and clinical variables. Complications were recorded and statistical analysis, including multivariate regression analysis, was performed. Results: The TAS group underwent significantly more skin-sparing mastectomies, adjuvant chemotherapy/radiation and less direct-to-implant reconstruction than the CHG group. The CHG group experienced a significantly lower incidence of total complications $(22.4 \%$ vs. $31.8 \%, \mathrm{p}=0.006)$, minor complications $(8.7 \%$ vs. $16.5 \%, \mathrm{p}=0.003)$, infection $(6.4 \%$ vs. $12.7 \%, \mathrm{p}=0.006)$ and seroma $(2.6 \%$ vs. $6.9 \%, \mathrm{p}=0.011)$. There was a significantly increased rate of delayed wound healing in the CHG group.
\end{abstract}


Multivariate analysis showed that the use of CHG solution significantly decreased the odds of any complication by 1.6 -fold (OR $0.637,95 \%$ CI 0.414 0.977 ) and the odds of infection by 2.4 -fold (OR 0.420, 95\% CI $0.218-0.809$ ). There were no statistically significant differences in rates of capsular contracture or other complications. Conclusions: The use of CHG as a pocket irrigant in post-mastectomy breast reconstruction is a reasonable alternative to other solutions, in efforts to minimize prosthetic based complications.

\section{Keywords}

Breast Reconstruction, Irrigation, Chlorohexidine

\section{Introduction}

Significant advances in breast cancer screening and management over the past thirty years have led to marked improvements in survivability after diagnosis and quality of life following oncologic treatment. According to the National Cancer Institute Surveillance, Epidemiology and End Results (SEER) database, 5-year overall survival for female patients with breast cancer from 2008-2014 was $89.7 \%$ [1]. Of the $62 \%$ of patients with localized disease and $31 \%$ of patients with regional disease, there was a $98.7 \%$ and $85.3 \%$ 5-year survival rate, respectively. These favorable statistics have paralleled global trends toward breast conserving therapy for patients with localized disease and contralateral mastectomy in patients requiring oncologic mastectomy in order to achieve an optimal reconstructive outcome. As a result, there has been a marked increase in the number of patients seeking breast reconstruction, demonstrated by a $62 \%$ increase in the rate of reconstructive procedures after mastectomy from 2009-2014 in one study [2].

The vast majority of breast reconstructive procedures following mastectomy are prosthetic-based. Of the 106,295 reconstructive breast procedures performed in the United States in 2017, 81.8\% utilized tissue expanders (TE) or single-stage direct-to-implant (DTI) reconstruction [3]. According to a recent report of 67,450 patients undergoing mastectomy for breast cancer from 2005-2014, the percentage of patients undergoing reconstruction has increased from $26.94 \%$ in 2005 to $43.30 \%$ in 2014, with a significant increase in the use of TE-based implantation from $15.54 \%$ to $33.30 \%$ [4]. The use of prosthetic materials for breast reconstruction, however, presents the potential for complications associated with synthetic and foreign materials implanted in vivo [5]-[10]. One of the most important modifiable factors to decrease the rate of complications is a reduction of the bacterial load within the breast pocket prior to implant placement, which has prompted the development of various additions to standard antiseptic technique [5] [11]-[16]. One important technique reported in the literature is the use of surgical site irrigation within the breast pocket [14] [16] [17] [18]. While there are multiple previous reports demonstrating decreased complications when using 
triple-antibiotic or povidone-iodine solutions, there has been a push for readyto-use off-the-shelf products. The purpose of this study is to investigate the difference in outcomes following implant-based breast reconstruction (IBBR) using triple-antibiotic (TAS) versus low-concentration chlorhexidine gluconate (CHG) solutions.

\section{Methods}

After obtaining Institutional Review Board approval, we performed an analysis of a prospectively maintained database of patients undergoing immediate implant-based breast reconstruction (IBBR) with either tissue-expanders or direct to implants between January 2008 and September 2017 at Emory University Hospital. All surgeries were performed by one of the two senior authors (GC and AL). Inclusion criteria included patients undergoing immediate implant-based reconstruction, no previous breast reconstruction or augmentation, use of either triple-antibiotic solution (50,000 IU bacitracin, $1 \mathrm{~g}$ cefazolin, $80 \mathrm{mg}$ gentamicin in $500 \mathrm{~mL}$ of normal saline) or Irrisept ${ }^{\oplus}(0.05 \%$ chlorhexidine gluconate in sterile water, Irrimax Corp, Lawrenceville, GA, USA), and complete medical records. Patients undergoing autologous or delayed implant-based breast reconstruction, no record of implant pocket irrigation, follow-up less than 30 days, or incomplete medical records were excluded from analysis. Analysis was performed on a per-breast basis.

Demographic and clinical characteristics were recorded, including age, BMI, history of smoking, diabetes and hypertension. Surgical intervention, including mastectomy type (skin-sparing mastectomy, nipple-sparing mastectomy or modified radical mastectomy), laterality (unilateral vs. bilateral), management of the axillary lymph node basin (sentinel lymph node biopsy vs. axillary lymph node dissection), and breast reconstruction (tissue expander, latissimus myocutaneous flap + tissue expander, or direct implant) were recorded. The use of neoadjuvant and/or adjuvant chemotherapy and radiation was also analyzed.

The primary outcome of interest was the incidence of postoperative complications. Complications were further categorized as delayed wound healing (superficial incisional dehiscence requiring serial dressing changes, office debridement and/or operative revision), infection (requiring oral or intravenous antibiotics or return to the operating room for incision and drainage), mastectomy skin flap necrosis (requiring serial dressing changes and/or debridement), implant/expander exposure, implant/expander failure, capsular contracture (Baker Grade III or IV), hematoma (requiring drainage in the operating room), seroma (requiring clinic or operating room aspiration/drainage), and fat necrosis. Major complications were those requiring hospital re-admission or return to the operating room. All other complications were recorded as minor.

Comparisons were made between demographic and clinical variables. The Mann-Whitney U-Test, Pearson chi-square, Fisher's exact, student's t-test were used as appropriate to compare outcomes between breast irrigation solution 
groups. Variables determined to be significantly different on univariate analysis that were included in the multivariate logistic regression model if $>50$ cases were present. All statistical analysis was conducted using the $\mathrm{IBM}^{\otimes}$ SPSS ${ }^{\oplus}$ Statistics 24.0 (IBM Corp., Armonk, N.Y.). All statistical tests used a 0.05 alpha level.

\section{Results}

During the 10-year study period, 1900 women underwent breast reconstruction following mastectomy. Of these, 690 subjects had sufficient data to be included for analysis. Data were calculated for the entire study group (Table 1) and then compared between the triple antibiotic solution (TAS) cohort and low-concentration chlorhexidine gluconate ( $\mathrm{CHG}$ ) cohort. The average follow up time was 854 days (range: 30 - 3550), with a mean of 36.1 months in the TAS group and mean of 18.8 months in the CHG group ( $\mathrm{p}<0.0001)$.

The average age of women undergoing breast reconstruction was 50.8 years (range: 26.9 - 82.1). The average BMI was $25.4 \mathrm{~kg} / \mathrm{m}^{2}$ (range: 14.0 - 44.7). Comorbidities included hypertension ( $\mathrm{n}=193,27.8 \%)$, diabetes $(\mathrm{n}=44,6.3 \%)$, and smoking history $(\mathrm{n}=27,3.9 \%)$. The CHG group was older (52.1 years vs. 49.7 years, $\mathrm{p}=0.007)$ and had a higher number of patients with hypertension $(\mathrm{n}=$ $115(33.4 \%)$ vs. $\mathrm{n}=78(22.5 \%), \mathrm{p}=0.002)$ when compared to the TAS group.

For the entire patient population, skin-sparing mastectomy (SSM) was performed most often ( $\mathrm{n}=482,70.1 \%$ ), followed by nipple-sparing mastectomy (NSM) $(\mathrm{n}=199,28.9 \%)$ and modified radical mastectomy (MRM) $(\mathrm{n}=7,1.0 \%)$. However, SSM was more prevalent in the TAS group $(\mathrm{n}=288(83.7 \%)$ vs. $\mathrm{n}=$ $194(56.4 \%)$ ), and NSM and MRM were more prevalent in the CHG group ( $\mathrm{n}=$ $145(42.2 \%)$ vs. $\mathrm{n}=54(15.7 \%)$ and $\mathrm{n}=5(1.5 \%)$ vs. $2(0.6 \%)$, respectively) ( $\mathrm{p}<$ 0.0001 ). Bilateral mastectomy was performed in $74.1 \%$ of cases and axillary lymph node dissection occurred in $25.7 \%$. There was no statistically significant difference in laterality or axillary lymph node management between the groups ( $\mathrm{p}=0.258$ and $\mathrm{p}=0.067$, respectively).

281 subjects (40.5\%) received chemotherapy and 168 (24.2\%) underwent radiation therapy. 164 subjects (47.4\%) in the TAS group underwent chemotherapy, while 115 patients $(33.4 \%)$ in the CHG group did ( $<<0.0001$ ). Similarly, 99 subjects $(28.6 \%)$ in the TAS group underwent radiation therapy, while 69 $(20.1 \%)$ in the CHG group did ( $\mathrm{p}=0.010)$.

All subjects had a prosthetic-based reconstruction with either tissue expander (TE) or direct-to-implant (DTI). Overall, TEs were used in 537 cases (77.8\%), DTI in 122 cases (17.7\%) and latissimus flap + TE in 31 cases (4.5\%). Patients in the TAS group had a higher percentage of women undergoing TE-based reconstruction compared to the CHG group $(\mathrm{n}=317(91.6 \%)$ vs. $\mathrm{n}=220(64.0 \%), \mathrm{p}<$ 0.0001); while DTI-based reconstruction occurred at a higher rate in the CHG group $(\mathrm{n}=93(27.0 \%)$ vs. $\mathrm{n}=29(8.4 \%), \mathrm{p}<0.0001)$. All patients who underwent latissimus flap + TE were in the CHG group. Use of acellular dermal matrix was similar between both groups: 194 (56.6\%) in the TAS group and 174 (50.6\%) in the CHG group. 
Table 1. Differences in clinical characteristics, oncologic treatment and complications in triple antibiotic vs. irrisept groups.

\begin{tabular}{|c|c|c|c|}
\hline Clinical characteristic & $\begin{array}{l}\text { Triple Antibiotic } \\
\quad(\mathrm{n}=346)\end{array}$ & Irrisept $(\mathrm{n}=344)$ & $\mathrm{p}$ \\
\hline Age, mean years & 49.7 & 52.1 & 0.007 \\
\hline BMI, mean $\mathrm{kg} / \mathrm{m}^{2}$ & 25.3 & 25.6 & 0.402 \\
\hline Smoking history, n (\%) & $11(3.2)$ & $16(4.7)$ & 0.334 \\
\hline Diabetes mellitus, n (\%) & $23(6.6)$ & $21(6.1)$ & 0.876 \\
\hline Hypertension, n (\%) & $78(22.5)$ & $115(33.4)$ & 0.002 \\
\hline Mastectomy type, n (\%) & & & $<0.0001$ \\
\hline Modified radical & $2(0.6)$ & $5(1.5)$ & \\
\hline Skin-sparing & $288(83.7)$ & $194(56.4)$ & \\
\hline Nipple-sparing & $54(15.7)$ & $145(42.2)$ & \\
\hline Laterality, n (\%) & & & 0.258 \\
\hline Unilateral & $96(27.9)$ & $82(23.8)$ & \\
\hline Bilateral & $248(72.1)$ & $262(76.2)$ & \\
\hline \multicolumn{4}{|l|}{ Lymph node dissection, $\mathrm{n}(\%)$} \\
\hline Sentinel lymph node biopsy & $269(78.2)$ & $269(78.2)$ & 1.000 \\
\hline Axillary lymph node dissection & $100(29.1)$ & $78(22.7)$ & 0.067 \\
\hline \multicolumn{4}{|l|}{ Chemotherapy, n (\%) } \\
\hline Any & $164(47.4)$ & $115(33.4)$ & $<0.0001$ \\
\hline Neoadjuvant & $82(23.7)$ & $79(23.0)$ & 0.857 \\
\hline Adjuvant & $98(28.3)$ & $55(16.0)$ & $<0.0001$ \\
\hline \multicolumn{4}{|l|}{ Radiotherapy, n (\%) } \\
\hline Any & $99(28.6)$ & $69(20.1)$ & 0.010 \\
\hline Neoadjuvant & $4(1.2)$ & $15(4.4)$ & 0.013 \\
\hline Adjuvant & $98(28.3)$ & $56(16.3)$ & $<0.0001$ \\
\hline Primary Reconstruction, $\mathrm{n}(\%)$ & & & $<0.0001$ \\
\hline Tissue expander & $317(91.6)$ & $220(64.0)$ & \\
\hline Direct-to-implant & $29(8.4)$ & $93(27.0)$ & \\
\hline Latissimus flap/expander & $0(0.0)$ & $31(9.0)$ & \\
\hline Use of acellular dermal matrix & $194(56.6)$ & $174(50.6)$ & 1.000 \\
\hline \multicolumn{4}{|l|}{ Complications, $\mathbf{n}(\%)$} \\
\hline Any complication & $110(31.8)$ & $77(22.4)$ & 0.006 \\
\hline Ipsilateral (to cancer side) & $81(23.4)$ & $59(17.2)$ & 0.047 \\
\hline Contralateral (to cancer side) & $32(9.2)$ & $19(5.5)$ & 0.080 \\
\hline Major complication & $69(19.9)$ & $55(16.0)$ & 0.198 \\
\hline Minor complication & $57(16.5)$ & $30(8.7)$ & 0.003 \\
\hline Delayed wound healing & $1(0.3)$ & $10(2.9)$ & 0.006 \\
\hline
\end{tabular}




\section{Continued}

\begin{tabular}{cccc}
\hline Infection & $44(12.7)$ & $22(6.4)$ & 0.006 \\
Mastectomy skin flap necrosis & $14(4.0)$ & $13(3.8)$ & 1.000 \\
Implant exposure & $3(0.9)$ & $9(2.6)$ & 0.089 \\
Implant failure & $28(8.1)$ & $16(4.7)$ & 0.086 \\
Capsular contracture & $28(8.1)$ & $16(4.7)$ & 0.086 \\
Hematoma & $11(3.2)$ & $15(4.4)$ & 0.432 \\
Seroma & $24(6.9)$ & $9(2.6)$ & 0.011 \\
Fat necrosis & $0(0.0)$ & $1(0.3)$ & 0.499 \\
Follow-up Time, months & 36.1 & 18.8 & $<0.0001$ \\
\hline
\end{tabular}

There were a total of 188 complications, 124 of which were considered major. Patients in the TAS group were more likely to experience a complication than patients in the CHG group ( $\mathrm{n}=110(31.8 \%)$ vs. $\mathrm{n}=77(22.4 \%), \mathrm{p}=0.006)$. Higher rates of complication following reconstruction were observed in the TAS group on the ipsilateral side to cancer resection $(\mathrm{n}=81(23.4 \%)$ vs. $\mathrm{n}=59$ (17.2\%), $\mathrm{p}=0.047)$ and in minor complications $(\mathrm{n}=57(16.5 \%)$ vs. $30(8.7 \%), \mathrm{p}$ $=0.003)$. Compared with the CHG group, the TAS group experienced a higher rate of infections ( $\mathrm{n}=44(12.7 \%)$ vs. $\mathrm{n}=22(6.4 \%), \mathrm{p}=0.006)$ and seromas $(\mathrm{n}=$ $24(6.9 \%)$ vs. $\mathrm{n}=9(2.6 \%), \mathrm{p}=0.011)$. Conversely, there was a higher rate of delayed wound healing in the CHG group $(\mathrm{n}=10(2.9 \%)$ vs. $\mathrm{n}=1(0.3 \%), \mathrm{p}=$ 0.003). On multivariate analysis, the odds of having any complication were 1.6-fold lower in the CHG group (OR $0.637,95 \%$ CI 0.414 - 0.977) and the odds of infection were 2.4 -fold lower (OR $0.420,95 \%$ CI 0.218 - 0.809). There were no statistically significant differences in major complications, complications on the contralateral reconstruction side, mastectomy skin flap necrosis, implant exposure, implant failure, capsular contracture, hematoma formation or fat necrosis.

\section{Discussion}

In 2017, more than 80,000 tissue expander or direct-to-implant reconstructive breast procedures were performed [3], representing the vast majority of breast reconstruction following mastectomy. Given the predominance of this technique and the use of synthetic materials, the prevention of complications has been a primary goal of surgeons performing prosthetic-based breast reconstruction. More specifically, reducing infection and capsular contracture has been of particular interest, as these pose the potential for devastating sequelae such as implant loss and the need for increasingly complex secondary reconstructions. While capsular contracture is a poorly understood phenomenon, there is a general consensus that subclinical infection and the development of peri-prosthetic biofilm contribute significantly [19] [20]. Thus, a significant body of literature has been produced in the pursuit of improved techniques that significantly diminish the potential for bacterial seeding of breast implants and tissue expanders 
in order to decrease rates of major complications [13] [17] [21].

Efforts to reduce the potential for bacterial seeding of breast implants include skin antiseptic preparation, soaking of the implant in an antibacterial solution, irrigating the breast pocket with an antibacterial solution, using a "no-touch" technique to introduce the breast implant into the breast pocket, and utilizing perioperative antibiotics. One national survey by Gowda et al. demonstrated that a majority of surgeons were using chlorhexidine to prepare the skin, soaking the implant in triple antibiotic solution and irrigating the breast pocket with povidone-iodine solution prior to implantation, then using a no-touch technique for implantation, and subsequently using first-generation cephalosporins until drains were removed [16]. Of these measures, it appears that breast pocket management (i.e. decreasing the local microbiome) has more of a protective effect than systemic antimicrobial prophylaxis. There has been increasing evidence that oral and parenteral perioperative antibiotics beyond 24 hours have limited efficacy in preventing implant-associated infections [21] [22]. Furthermore, there is literature to support that the administration of prolonged antibiotics has the potential to give rise to drug-resistant organisms that are more difficult to eradicate if an attempt at implant salvage becomes necessary [23] [24]. Thus, the American Society of Plastic Surgery has clear guidelines stating that patients undergoing breast reconstruction with tissue expanders or implants should be administered a pre-operative antibiotic, but all antibiotics should be discontinued within 24 hours of the operation unless a surgical drain is present [12].

Local bacterial eradication and the creation of a microbe-free implant pocket are essential to preventing complications in prosthetic-based breast reconstruction. Breast pocket irrigation using antimicrobial solutions remains one of the important ways by which this can be achieved. The most common irrigation solutions include Adams' triple antibiotic solution (TAS; 50,000 IU bacitracin, $1 \mathrm{~g}$ cefazolin, $80 \mathrm{mg}$ gentamicin in $500 \mathrm{~mL}$ of normal saline) and povidone-iodine (P-I). While TAS has been widely used by plastic surgeons, there is little and conflicting evidence demonstrating its efficacy in preventing complications. For example, Adams and colleagues showed that TAS decreases bacterial populations responsible for peri-prosthetic infections and leads to reduced rates of capsular contracture for breast reconstruction [25] [26]; however, subsequent contrasting studies question the effect of TAS to reduce the rate of capsular contracture [27] [28]. In comparison to TAS, P-I reduces infection risk as an antiseptic solution. Review of current literature demonstrates the effectiveness of P-I to reduce subclinical infections and capsular contracture [29] [30] [31] [32]. Additional studies have advocated for the use of breast pocket irrigation containing both antibiotic solution and P-I [17].

In 2000, the Food and Drug Administration (FDA) issued a ban on the use of Betadine for breast implants given a concern for delamination of shell integrity and the potential for implant rupture [33]. Although multiple studies have suggested this to be exceedingly unlikely [29] [30] [32] [34] [35], the proper use of Betadine to irrigate the breast pocket and resultant implant complications car- 
ries the risk of legal implications [33]. For this reason, the immediate washout of Betadine irrigation with saline has been recommended, but this practice limits the antimicrobial effectiveness of Betadine [14] [33].

Another alternative to the above includes an antiseptic irrigation solution containing chlorhexidine gluconate ( $\mathrm{CHG}$ ), which has been shown to be an effective and relatively cheap antimicrobial that has both bacteriostatic and bactericidal effects against a wide range bacteria and fungi. CHG has been shown to be more effective in preventing surgical site infections than skin preparation with traditional povidone-iodine [36] [37] [38]. While there is no current clinical literature demonstrating the use of CHG as a breast pocket irrigation solution, there have been in vitro studies that establish superiority of CHG to P-I to eliminate the bacteria implicated in peri-prosthetic infection and the development of biofilms at shorter exposure times [18] [39]. This pre-clinical data thus supports CHG solution as a clinically viable option to decrease bacterial burden in the breast pocket without sacrificing operative time. Of note, there have been reports of rare allergic reactions to chlorhexidine-containing solutions which need to be taken into consideration [40], however, in lower concentrations (i.e. < $0.05 \%)$, this is less of an issue [41].

With this background, the purpose of our study was to compare the incidence of postoperative complications following implant-based breast reconstruction using either TAS or CHG for breast pocket irrigation. Our results reflect the pre-clinical studies in that patients undergoing breast pocket irrigation with CHG had significantly less risk of infection (6.4\% vs. $12.7 \%, \mathrm{p}=0.006)$, seroma ( $2.6 \%$ vs. $6.9 \%, \mathrm{p}=0.011)$, minor complications $(8.7 \%$ vs. $16.5 \%, \mathrm{p}=0.003)$ and total complications $(22.4 \%$ vs. $31.8 \%, \mathrm{p}=0.006)$ when compared to patients with TAS breast pocket irrigation. Furthermore, implant failure (4.7\% vs. $8.1 \%, \mathrm{p}=$ $0.086)$ and capsular contracture $(4.7 \%$ vs. $8.1 \%, \mathrm{p}=0.086)$ had trends towards significantly less incidence in the CHG group. We expect that with a larger sample size, the trends towards less implant failure and less capsular contracture would become statistically significant. These data suggest that CHG promotes sufficient breast pocket antisepsis and the potential for fewer complications when compared to TAS. Given the differences in patient follow-up and anecdotal improvements in mastectomy skin flap quality for our study population, these results should rather be regarded as CHG being a non-inferior alternative to TAS.

While our data are promising, it is important to note some potential complicating and limiting factors. First of all, this was a retrospective post-hoc analysis. Although variation in technique was significantly controlled by the fact that two surgeons performed all of the operations, there may have been small changes that led to improved outcomes in the CHG group that may not be attributed to the use of CHG alone. One noticeable change in technique was the increased use of direct-to-implant practice rather than staged tissue-expander reconstruction in the CHG group (27.0\% vs. $8.4 \%)$. Clearly, single-staged reconstruction has less potential for bacterial seeding of the breast implant, which could potentially 
muddle our results. Perhaps more importantly, however, is the fact that patients in the CHG group underwent chemotherapy and radiation at significantly decreased rates when compared to the TAS group (33.4\% vs. $47.4 \%, \mathrm{p}<0.0001$; and $20.1 \%$ vs. $28.6, p=0.010$, respectively). The increased rates of neoadjuvant and adjuvant therapy are important potential confounders as these are known to contribute to suboptimal healing; however, when controlling for these on multivariate analysis, our results remained valid with 2.4 -fold decreased odds of infection, 2.8-fold decreased odds of minor complications and 1.6-fold decreased odds of any complication in the CHG group. Interestingly, the one complication that was increased in the CHG group was delayed wound healing (2.9\% vs. $0.3 \%$, $\mathrm{p}=0.006$ ). Finally, it is important to note that this was a relatively small single-center study with 690 subjects analyzed in a per-breast fashion. Thus, while our results do suggest improved outcomes with CHG compared to TAS, it is important to perform larger, multi-center studies with multiple surgeons utilizing different implants and techniques to obtain more generalizable data.

\section{Conclusion}

This was a retrospective review of a prospectively maintained database comparing triple antibiotic solution to low-concentration chlorhexidine gluconate for breast pocket irrigation in implant-based breast reconstruction. We found that the CHG group experienced significantly less overall and minor complications, had fewer infections and developed fewer seromas. There was no statistically significant difference in other complications analyzed; however, there was a trend toward decreased implant failure and capsular contracture in the $\mathrm{CHG}$ group as well. Future work with larger datasets is needed to confirm and expand these findings.

\section{Financial Disclosures}

None of the authors has a financial interest in any of the products, devices, or drugs mentioned in this manuscript.

\section{Author Contribution}

All of the aforementioned authors were involved in the acquisition, analysis and/or interpretation of data, drafting and/or revision of the manuscript, gave final approval for submission of the manuscript, and agree to be accountable for the data presented in the submitted manuscript.

\section{Conflicts of Interest}

The authors declare no conflicts of interest regarding the publication of this paper.

\section{References}

[1] Cancer Stat Facts: Female Breast Cancer (2018) National Cancer Institute Surveil- 
lance, Epidemiology and End Results (SEER) Program.

[2] Miller, A.M., Steiner, C.A., Barrett, M.L., Fingar, K.R. and Elixhauser, A. (2006) Breast Reconstruction Surgery for Mastectomy in Hospital Inpatient and Ambulatory Settings, 2009-2014: Statistical Brief \#228. Healthcare Cost and Utilization Project (HCUP) Statistical Briefs. Rockville.

[3] American Society of Plastic Surgeons Plastic Surgery Statistics Report (2017) ASPS National Clearinghouse of Plastic Surgery Procedural Statistics, 2017.

[4] Ilonzo, N., Tsang, A., Tsantes, S., Estabrook, A. and Thu Ma, A.M. (2017) Breast Reconstruction after Mastectomy: A Ten-Year Analysis of Trends and Immediate Postoperative Outcomes. Breast, 32, 7-12. https://doi.org/10.1016/j.breast.2016.11.023

[5] Cohen, J.B., Carroll, C., Tenenbaum, M.M. and Myckatyn, T.M. (2015) Breast Implant-Associated Infections: The Role of the National Surgical Quality Improvement Program and the Local Microbiome. Plastic and Reconstructive Surgery, 136, 921-929. https://doi.org/10.1097/PRS.0000000000001682

[6] Forster, N.A., Kunzi, W. and Giovanoli, P. (2013) The Reoperation Cascade after Breast Augmentation with Implants: What the Patient Needs to Know. Journal of Plastic, Reconstructive \& Aesthetic Surgery, 66, 313-322. https://doi.org/10.1016/j.bjps.2012.09.033

[7] Hidalgo, D.A. and Sinno, S. (2016) Current Trends and Controversies in Breast Augmentation. Plastic and Reconstructive Surgery, 137, 1142-1150.

https://doi.org/10.1097/01.prs.0000481110.31939.e4

[8] Ota, D., Fukuuchi, A., Iwahira, Y., et al. (2016) Identification of Complications in Mastectomy with Immediate Reconstruction Using Tissue Expanders and Permanent Implants for Breast Cancer Patients. Breast Cancer, 23, 400-406. https://doi.org/10.1007/s12282-014-0577-4

[9] Ozturk, C.N., Ozturk, C., Soucise, A., et al. (2018) Expander/Implant Removal after Breast Reconstruction: Analysis of Risk Factors and Timeline. Aesthetic Plastic Surgery, 42, 64-72. https://doi.org/10.1007/s00266-017-1031-8

[10] Song, J.H., Kim, Y.S., Jung, B.K., et al. (2017) Salvage of Infected Breast Implants. Archives of Plastic Surgery, 44, 516-522. https://doi.org/10.5999/aps.2017.01025

[11] Adams, W.P., Rios, J.L. and Smith, S.J. (2006) Enhancing Patient Outcomes in Aesthetic and Reconstructive Breast Surgery Using Triple Antibiotic Breast Irrigation: Six-Year Prospective Clinical Study. Plastic and Reconstructive Surgery, 117, 30-36.

[12] Alderman, A., Gutowski, K., Ahuja, A. and Gray, D. (2014) Postmastectomy Expander Implant Breast Reconstruction Guideline Work G. ASPS Clinical Practice Guideline Summary on Breast Reconstruction with Expanders and Implants. Plastic and Reconstructive Surgery, 134, 648e-655e.

https://doi.org/10.1097/PRS.0000000000000541

[13] Araco, A., Gravante, G., Araco, F., Delogu, D., Cervelli, V. and Walgenbach, K. (2007) Infections of Breast Implants in Aesthetic Breast Augmentations: A Single-Center Review of 3,002 Patients. Aesthetic Plastic Surgery, 31, 325-329. https://doi.org/10.1007/s00266-006-0156-y

[14] Craft, R.O., Damjanovic, B. and Colwell, A.S. (2012) Evidence-Based Protocol for Infection Control in Immediate Implant-Based Breast Reconstruction. Annals of Plastic Surgery, 69, 446-450. https://doi.org/10.1097/SAP.0b013e31824a215a

[15] Galdiero, M., Larocca, F., Iovene, M.R., et al. (2018) Microbial Evaluation in Capsular Contracture of Breast Implants. Plastic and Reconstructive Surgery, 141, 23-30. https://doi.org/10.1097/PRS.0000000000003915 
[16] Gowda, A.U., Chopra, K., Brown, E.N., Slezak, S. and Rasko, Y. (2017) Preventing Breast Implant Contamination in Breast Reconstruction: A National Survey of Current Practice. Annals of Plastic Surgery, 78, 153-156.

https://doi.org/10.1097/SAP.0000000000000822

[17] Giordano, S., Peltoniemi, H., Lilius, P. and Salmi, A. (2013) Povidone-Iodine Combined with Antibiotic Topical Irrigation to Reduce Capsular Contracture in Cosmetic Breast Augmentation: A Comparative Study. Aesthetic Surgery Journal, 33, 675-680. https://doi.org/10.1177/1090820X13491490

[18] Zhadan, O. and Becker, H. (2018) Surgical Site Irrigation in Plastic Surgery. Aesthetic Surgery Journal, 38, 265-273. https://doi.org/10.1093/asj/sjx171

[19] Rieger, U.M., Mesina, J., Kalbermatten, D.F., et al. (2013) Bacterial Biofilms and Capsular Contracture in Patients with Breast Implants. British Journal of Surgery, 100, 768-774. https://doi.org/10.1002/bjs.9084

[20] Bartsich, S., Ascherman, J.A., Whittier, S., Yao, C.A. and Rohde, C. (2011) The Breast: A Clean-Contaminated Surgical Site. Aesthetic Surgery Journal, 31, 802-806. https://doi.org/10.1177/1090820X11417428

[21] Viola, G.M., Raad, I.I. and Rolston, K.V. (2014) Breast Tissue Expander-Related Infections: Perioperative Antimicrobial Regimens. Infection Control \& Hospital Epidemiology, 35, 75-81. https://doi.org/10.1086/674390

[22] Phillips, B.T., Bishawi, M., Dagum, A.B., Khan, S.U. and Bui, D.T. (2013) A Systematic Review of Antibiotic Use and Infection in Breast Reconstruction: What Is the Evidence? Plastic and Reconstructive Surgery, 131, 1-13.

[23] Ranganathan, K., Sears, E.D., Zhong, L., et al. (2018) Antibiotic Prophylaxis after Immediate Breast Reconstruction: The Reality of Its Efficacy. Plastic and Reconstructive Surgery, 141, 865-877. https://doi.org/10.1097/PRS.0000000000004204

[24] McCullough, M.C., Chu, C.K., Duggal, C.S., Losken, A. and Carlson, G.W. (2016) Antibiotic Prophylaxis and Resistance in Surgical Site Infection after Immediate Tissue Expander Reconstruction of the Breast. Annals of Plastic Surgery, 77, 501-505. https://doi.org/10.1097/SAP.0000000000000275

[25] Adams, W.P., Conner, W.C., Barton, F.E. and Rohrich, R.J. (2000) Optimizing Breast Pocket Irrigation: An in Vitro Study and Clinical Implications. Plastic and Reconstructive Surgery, 105, 9-43. https://doi.org/10.1097/00006534-200001000-00053

[26] Adams, W.P., Rios, J.L. and Smith, S.J. (2006) Enhancing Patient Outcomes in Aesthetic and Reconstructive Breast Surgery Using Triple Antibiotic Breast Irrigation: Six-Year Prospective Clinical Study. Plastic and Reconstructive Surgery, 118, 46S-52S. https://doi.org/10.1097/01.prs.0000185671.51993.7e

[27] Drinane, J.J., Chowdhry, T., Pham, T.H. and Ritter, E. (2017) Examining the Role of Antimicrobial Irrigation and Capsular Contracture: A Systematic Review and Meta-Analysis. Annals of Plastic Surgery, 79, 107-114. https://doi.org/10.1097/SAP.0000000000001134

[28] Drinane, J.J., Kortes, M.J., Bergman, R.S. and Folkers, B.L. (2016) Evaluation of Antibiotic Irrigation versus Saline Irrigation in Reducing the Long-Term Incidence and Severity of Capsular Contraction after Primary Augmentation Mammoplasty. Annals of Plastic Surgery, 77, 32-36. https://doi.org/10.1097/SAP.0000000000000302

[29] Burkhardt, B.R. and Demas, C.P. (1994) The Effect of Siltex Texturing and Povidone-Iodine Irrigation on Capsular Contracture around Saline Inflatable Breast Implants. Plastic and Reconstructive Surgery, 93, 123-128. 
https://doi.org/10.1097/00006534-199401000-00018

[30] Burkhardt, B.R. and Eades, E. (1995) The Effect of Biocell Texturing and Povidone-Iodine Irrigation on Capsular Contracture around Saline-Inflatable Breast Implants. Plastic and Reconstructive Surgery, 96, 1317-1325.

https://doi.org/10.1097/00006534-199511000-00013

[31] McGuire, P., Reisman, N.R. and Murphy, D.K. (2017) Risk Factor Analysis for Capsular Contracture, Malposition, and Late Seroma in Subjects Receiving Natrelle 410 Form-Stable Silicone Breast Implants. Plastic and Reconstructive Surgery, 139, 1-9. https://doi.org/10.1097/PRS.0000000000002837

[32] Yalanis, G.C., Liu, E.W. and Cheng, H.T. (2015) Efficacy and Safety of Povidone-Iodine Irrigation in Reducing the Risk of Capsular Contracture in Aesthetic Breast Augmentation: A Systematic Review and Meta-Analysis. Plastic and Reconstructive Surgery, 136, 687-698. https://doi.org/10.1097/PRS.0000000000001576

[33] Wiener, T.C. (2013) Betadine and Breast Implants: An Update. Aesthetic Surgery Journal, 33, 615-617. https://doi.org/10.1177/1090820X13484036

[34] Wiener, T.C. (2007) The Role of Betadine Irrigation in Breast Augmentation. Plastic and Reconstructive Surgery, 119, 12-15. https://doi.org/10.1097/01.prs.0000251088.51675.a8

[35] Zambacos, G.J., Nguyen, D. and Morris, R.J. (2004) Effect of Povidone Iodine on Silicone Gel Breast Implants in Vitro: Implications for Clinical Practice. Plastic and Reconstructive Surgery, 114, 706-710. https://doi.org/10.1097/01.PRS.0000130972.59268.AA

[36] Darouiche, R.O., Wall, M.J., Itani, K.M., et al. (2010) Chlorhexidine-Alcohol versus Povidone-Iodine for Surgical-Site Antisepsis. The New England Journal of Medicine, 362, 18-26. https://doi.org/10.1056/NEJMoa0810988

[37] Dumville, J.C., McFarlane, E., Edwards, P., Lipp, A., Holmes, A. and Liu, Z. (2015) Preoperative Skin Antiseptics for Preventing Surgical Wound Infections after Clean Surgery. Cochrane Database of Systematic Reviews, No. 4, CD003949. https://doi.org/10.1002/14651858.CD003949.pub4

[38] Edmiston, C.E., Bruden, B., Rucinski, M.C., Henen, C., Graham, M.B. and Lewis, B.L. (2013) Reducing the Risk of Surgical Site Infections: Does Chlorhexidine Gluconate Provide a Risk Reduction Benefit? American Journal of Infection Control, 41, S49-S55. https://doi.org/10.1016/j.ajic.2012.10.030

[39] Schmidt, K., Estes, C., McLaren, A. and Spangehl, M.J. (2018) Chlorhexidine Antiseptic Irrigation Eradicates Staphylococcus epidermidis from Biofilm: An in Vitro Study. Clinical Orthopaedics and Related Research, 476, 648-653. https://doi.org/10.1007/s11999.0000000000000052

[40] FDA (2017) FDA Drug Safety Communication: FDA Warns about Rare But Serious Allergic Reactions with the Skin Antiseptic Chlorhexidine Gluconate.

[41] Rose, M.A., Garcez, T., Savic, S. and Garvey, L.H. (2019) Chlorhexidine Allergy in the Perioperative Setting: A Narrative Review. British Journal of Anaesthesia, 123, e95-e103. https://doi.org/10.1016/j.bja.2019.01.033 